\title{
Safire : des avions au service de la recherche en environnement
}

\author{
Caroline Lamorthe', Agnès Borbon'2, Alfons Schwarzenboeck ${ }^{2}$, \\ Jean-Christophe Canonici ${ }^{1}$ \\ 1 Safire, Météo-France / CNRS / Cnes, Cugnaux \\ 2 Laboratoire de météorologie physique, Université Blaise-Pascal / CNRS, Aubière \\ jc.canonici@safire.fr
}

\section{Résumé}

Au travers du Service des avions français instrumentés pour la recherche en environnement (Safire) et de sa flotte, la France dispose d'une infrastructure publique de recherche qui met trois avions à disposition de la communauté scientifique pour des mesures en altitude. Sont installés à bord de ces avions, en cabine ou fixés sur le fuselage ou sous les ailes, des instruments choisis dans une vaste panoplie en fonction des paramètres physico-chimiques de l'atmosphère ou de la surface terrestre que les chercheurs veulent étudier. Après une revue des moyens, du fonctionnement de Safire et de l'organisation des campagnes de mesures, cet article présente les opérations aériennes et l'instrumentation de Safire, ainsi que le traitement des données. Enfin, deux exemples de campagnes expérimentales récentes impliquant des avions de Safire sont détaillés.

\section{Abstract}

\section{SAFIRE: aircraft for environmental research}

With its Safire fleet, France manages a public research infrastructure that supplies the scientific community with three aircraft for altitude measurements. A wide variety of instruments can be installed on the aircraft, either inside the cabin or on the fuselage or under the wings, according to the physico-chemical parameters of the atmosphere or the Earth's surface to be investigated. After a review of the facilities, the organization of SAFIRE and the management of experimental campaigns, we give an introduction to airborne operations, instrumentation and data processing. Finally two examples of experimental campaigns are described. e Service des avions français instrumentés pour la recherche en environnement (Safire) est une unité mixte de service du CNRS, de Météo-France et du Cnes : chercheurs et ingénieurs en sont les usagers pour mener à bien les expériences nécessitant des mesures aéroportées in situ ou par télédétection.

En France, plusieurs dizaines de laboratoires sont concernés par l'expérimentation scientifique aéroportée ${ }^{1}$. Nombre d'entre eux se reposent entièrement sur Safire pour les vols comme pour le processus d'installation instrumentale. Mais certains ont acheté ou conçu et développé en interne leurs propres instruments pour répondre à des besoins spécifiques. Ils ont ensuite recours à des prestataires, privés ou publics, dont Safire. Les principaux thèmes des expérimentations portent sur l'observation atmosphérique, océanographique et des surfaces continentales, la recherche et le développement aéronautique et le soutien aux missions spatiales.

De fait, Safire occupe une place particulière dans le paysage de la recherche aéroportée, avec ses trois avions ainsi qu'une large gamme d'instruments scientifiques. Cette infrastructure de recherche publique française est de portée internationale.

Tout chercheur français ayant un projet de recherche peut mettre à contribution Safire pour le réaliser sous réserve de le faire valider par le Comité scientifique et technique avions (CSTA) et de rassembler le financement nécessaire pour en couvrir les coûts.

1. Enquête effectuée en 2014 auprès de plus de 200 laboratoires par le CSTA.

\section{Une unité mixte de service}

En tant qu'unité mixte de services, Safire est gouverné par un comité de direction constitué de représentants du Cnes, de Météo-France et du CNRS (plus particulièrement de l'Insu, Institut national de sciences de l'Univers). Le comité prend l'avis de la communauté des chercheurs au travers du CSTA, composé d'experts et de représentants des principaux laboratoires utilisateurs de Safire.

Les tutelles financent le fonctionnement courant de l'unité. Une autre part, substantielle, des ressources de Safire provient des laboratoires ou organismes utilisateurs de ses services, en particulier via des projets financés par l'Agence nationale de la recherche, la Commission européenne ou encore des agences gouvernementales qui soutiennent souvent les utilisateurs de Safire.

Les agents de Safire, issus du CNRS et de Météo-France, sont majoritairement des ingénieurs ou des techniciens, spécialistes de techniques de mesures aéroportées. Des pilotes professionnels, des mécaniciens aéronautiques et du personnel support complètent le dispositif. La base de safire est située sur l'aéroport de Toulouse-Francazal (figure 1).

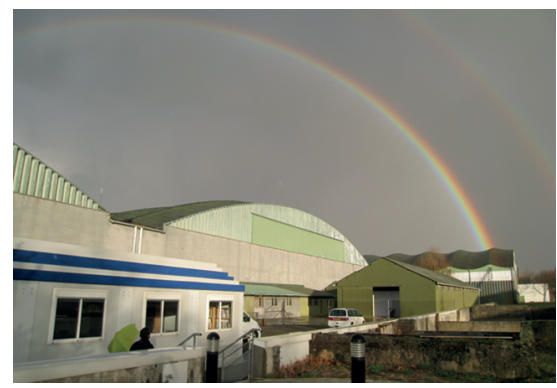

Figure 1. Locaux de Francazal. 


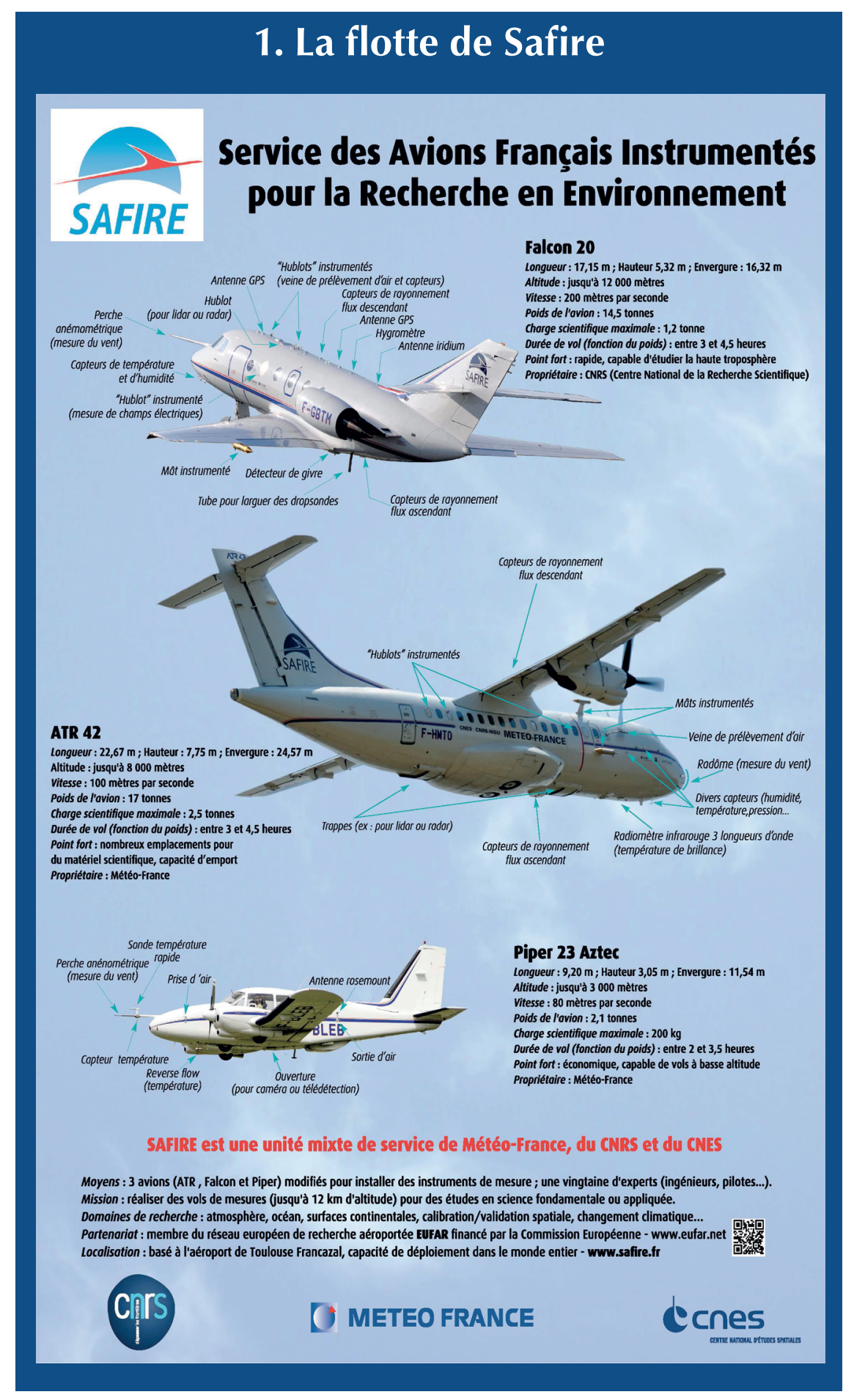

\section{Les avions de Safire}

L'ATR 42 est un bi-turbopropulseur qui peut voler jusqu'à 8000 mètres d'altitude. En raison de sa taille imposante d'avion commercial, avec une capacité d'emport de 2,5 tonnes de matériel, il permet à plusieurs laboratoires de collaborer en utilisant leurs propres instruments lors de vols mutualisés (encadré 1).

À l'origine biréacteur d'affaires, le Falcon 20 explore de plus hautes altitudes avec un plafond de $12000 \mathrm{~m}$.
Sa vitesse de croisière au moment de la mesure est de $200 \mathrm{~m} / \mathrm{s}$, soit deux fois plus élevée que celle de l'ATR : cela en fait l'avion le plus rapide de la flotte.

Le Piper Aztec, vétéran de la flotte avec particulièrement adapté aux mesures en basses couches. Ses caractéristiques lui permettent de faire des tests à bas coût et de voler là où des avions plus gros ne peuvent pas aller. Il a ainsi pu effectuer des survols d'agglomérations à basse altitude qui auraient été interdits à des 40 ans de service, est un petit bimoteur avions plus volumineux.

\section{Une vocation internationale dans une structure européenne}

Avec ses trois avions, Safire représente une des plus grosses flottes européennes dédiées à la recherche. Un tel dispositif reste un atout majeur pour soutenir la compétitivité des équipes françaises et garantir leur participation aux grandes campagnes internationales de mesure. Naturellement au service des chercheurs de notre pays, Safire fait aussi voler des scientifiques de toute nationalité sur tous les continents. En parallèle, la recherche aéroportée bénéficie de la complémentarité des vecteurs européens réunis au sein du consortium Eufar (European fleet for airborne research). Cette « initiative d'infrastructure intégrée (I3) » européenne permet notamment à tous les scientifiques européens, qu'ils disposent ou non d'un outil national, de bénéficier de vols scientifiques financés. Safire a réalisé plusieurs dizaines d'heures de vol dans ce cadre et, sous l'impulsion de ses tutelles, joue un rôle moteur dans ce mouvement européen.

Très prochainement, Eufar devrait se consolider en une fédération d'unités nationales au moyen d'une entité juridique commune.

\section{Une grande variété de thèmes de recherche}

Compte tenu de son histoire (encadré 2) et de ses liens forts avec Météo-France, il est naturel que le principal thème des recherches impliquant Safire porte sur l'observation de l'atmosphère. Les enjeux scientifiques autour de la météorologie et du climat nécessitent toujours plus d'observations des interactions entre dynamique, nuages, aérosols, chimie et rayonnement. L'avion reste un outil indispensable par son aptitude à pénétrer dans les phénomènes atmosphériques que les chercheurs souhaitent étudier. Les mesures in situ effectuées à partir d'avions de recherche classiques concernent essentiellement la troposphère, où l'on peut approcher la plupart des phénomènes météorologiques que ce soit dans la couche limite ou au sommet des nuages convectifs. La 


\section{80 ans d'aviation de recherche en environnement}

Les historiens rapportent que, dès ses balbutiements, la conquête de l'air par les avions a donné lieu à des expériences physiques sur l'atmosphère, tout comme les techniques d'exploration par cerf-volant et ballon-sonde qui ont contribué à la naissance de la météorologie moderne. Néanmoins, il faut attendre la mise au point d'avions légers et fiables pour que le Service météorologique français, l'ONM (Office national météorologique), se dote en 1936 et à l'initiative de l'ingénieur René Eyraud d'un motoplaneur (photo 2) et dont le devis sur papier pelure est pieusement conservé dans les archives de Safire...

Les reconnaissances météorologiques aériennes effectuées pendant la deuxième guerre mondiale ont fait gagner du galon aux avions de recherche : à partir de 1947, le CAEM (Centre aérien d'études météorologiques), ancêtre de Safire, et la SASM (Section d'avions de sondages météorologiques), unité militaire exploitant des aéronefs, collaborent en mêlant ingénieurs civils et moyens militaires.

À la fin des années 1960, la recherche amorce une nouvelle phase en se dotant de moyens aéroportés en propre. L'objectif est de mettre en place des méthodes éprouvées afin d'étudier I'atmosphère et les surfaces océaniques ou continentales à partir d'avions, en complément des données au sol.

Basé dans les installations du Centre d'essais en vol (CEV) de Brétignysur-Orge, l'équipe d'aviation météorologique du Service de

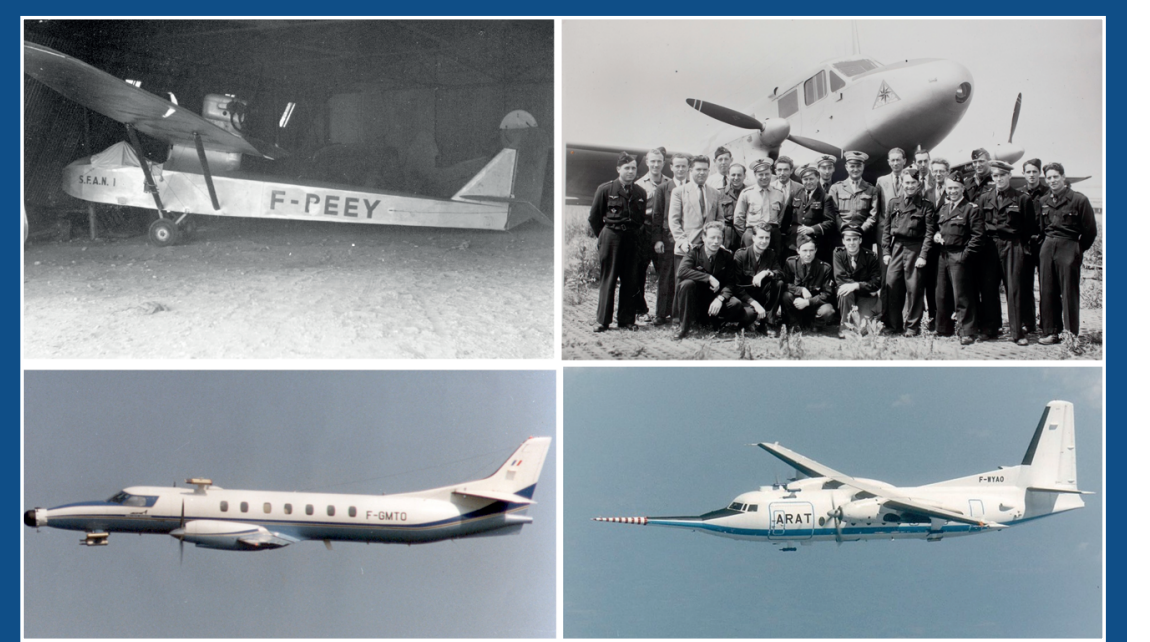

De gauche à droite et de haut en bas : motoplaneur SFAN 01 (ONM), Caudron Goéland (DMN) Merlin IV (DMN), ARAT (CNRS)

basse stratosphère peut également être sondée grâce aux moyens de télédétection embarqués, permettant une mesure instantanée sur l'ensemble de la colonne

recherche de la Météorologie nationale, I'EERM (Établissement d'études et de recherches météorologiques), achète avec le CNRS et la DGRST (Direction générale de la recherche scientifique et technique (ministère de la Défense)) en 1968 un avion léger Cessna. En 1986, l'EERM le remplace par un avion bi-turbo-propulseur, le Merlin IV, qui volera jusqu'en 2003. Cette structure continuera néanmoins à utiliser ponctuellement des avions plus gros ou volant plus haut appartenant au CEV, par exemple pour mesurer les champs $d^{\prime}$ ozone stratosphérique ou étudier les foudroiements des avions dans les orages (Chalon et al., 1998 ; Brenguier et Ancellet, 2003). De son côté, le CNRS achète son premier avion de recherche atmosphérique, un Aerocommander, puis investit avec l'aide financière du Cnes, de Météo-France et de l'IGN (Institut national de l'information géographique et forestière) dans un Fokker 27 lourdement modifié qui sera appelé Arat (Avion de recherche atmosphérique et de télédétection). Basé à Creil, il utilise les moyens de l'IGN (pilotes et maintenance) et lui emprunte parfois certains de ses avions comme le mythique Hurel-Dubois à aile " à grand allongement ».

La mutualisation des moyens de Météo-France, du CNRS et du Cnes prend sa pleine dimension avec la création en 2005 de Safire, visant à regrouper matériel, personnel et budget autour de trois avions dont deux nouveaux : le Falcon 20, propriété du CNRS, I'ATR 42 et le Piper Aztec appartenant à Météo-France. 


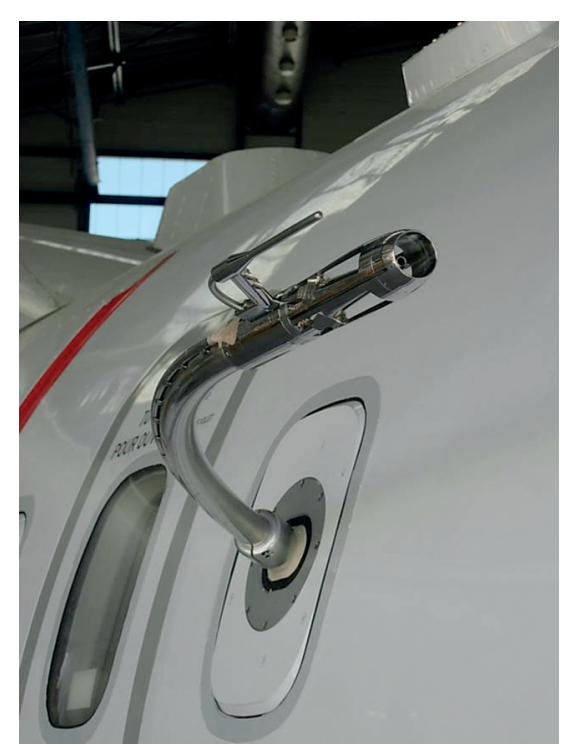

Figure 2. La veine communautaire échantillonne l'air de façon isocinétique pour une distribution vers plusieurs appareils de mesure.

l'expérience en temps réel en fonction des informations recueillies à bord (figure 3).

Il n'y a pas de passager dans ces avions classés en «travail aérien » : chacun à bord est membre de l'équipage et enregistré en tant que tel sur le manifeste de vol. Scientifiques et ingénieurs n'embarquent qu'après avoir satisfait aux exigences d'absence de contre-indications médicales et reçu une autorisation écrite de leurs

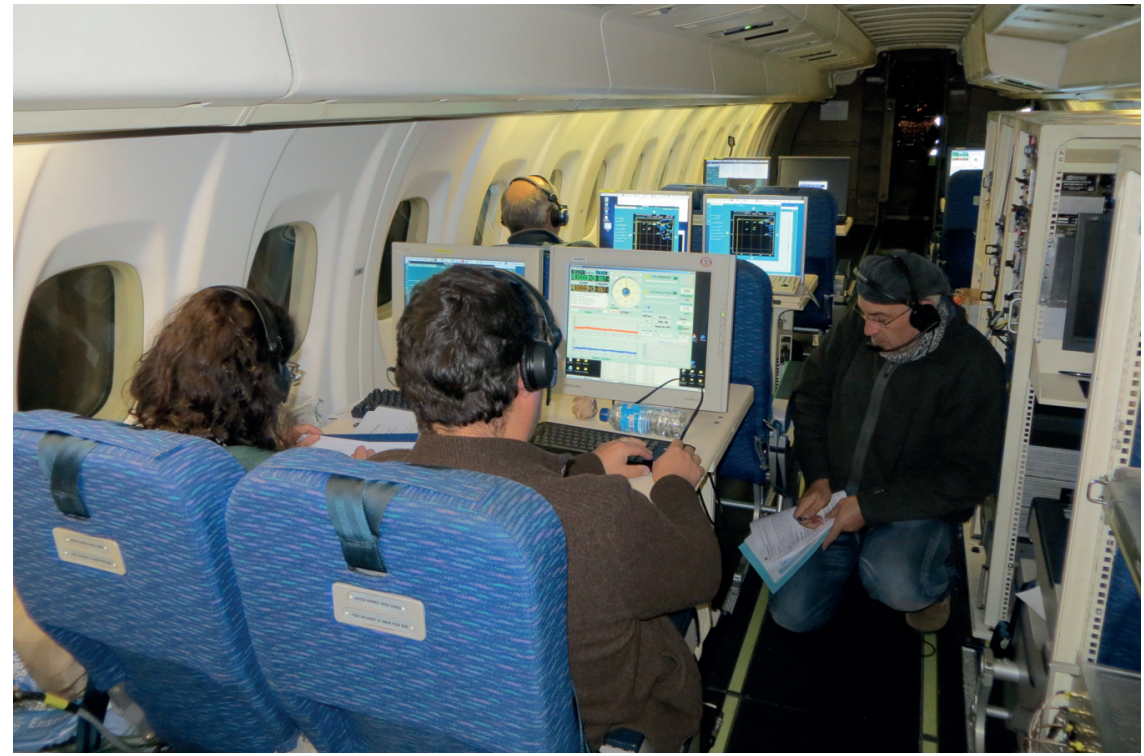

Figure 3. Scientifiques et ingénieurs d'essais dans l'ATR42 pendant la campagne Kuros en octobre 2015.

supérieurs hiérarchiques. On ne transige pas avec la sécurité dans le domaine très normé de l'aéronautique, surtout quand on étudie des phénomènes potentiellement dangereux pour l'avion comme la foudre ou la convection. Ainsi, on a du s'approcher des systèmes convectifs tout en évitant les dangers des orages pour étudier la taille et la densité des cristaux de glace à haute altitude lors de la campagne HAIC-HIWC (voir plus loin). De même, la mesure de flux entre l'océan

\section{Piloter un avion de recherche}

Parmi les 23 postes permanents de Safire, trois sont réservés à des pilotes professionnels. L'emploi est très spécifique, car il nécessite de concilier les contraintes classiques de l'aviation avec celles de la recherche, que ce soit lors des phases de préparation en amont des expériences, ou en vol durant celles-ci.

Rarement le plan de vol se déroule en reliant deux points par les routes aériennes classiques. Plusieurs mois avant l'expérience, les pilotes travaillent avec les scientifiques, puis négocient avec les autorités de contrôle aérien pour pouvoir effectuer des vols " atypiques", souvent en dehors des couloirs aériens et avec de fréquents changements de niveau de vol. Certaines trajectoires sont imprévisibles, par exemple quand on souhaite étudier des phénomènes en temps réel comme des pluies violentes ou des orages. D'autres exigent au contraire une grande précision, comme pour suivre la même trajectoire que la trace d'un satellite. Dans les deux cas, cela peut impliquer de couper plusieurs couloirs aériens. II faut alors demander longtemps à l'avance des autorisations spéciales et utiliser I'appui d'un contrôleur aérien dédié en évoluant parfois dans des zones normalement réservées au trafic militaire. En parallèle à ce travail discret mais chronophage de préparation opérationnelle des vols, les pilotes se livrent à un entraînement aérien poussé. Ils effectuent également une veille réglementaire et technique pour maintenir en conformité I'organisation des vols.

En vol, cette polyvalence des pilotes implique, par exemple, une double écoute par casques : par radio avec la tour de contrôle et par téléphone de bord avec les expérimentateurs dans la cabine. Un suivi très précis des conditions météorologiques environnantes est capital, non seulement pour assurer une gestion optimale du vol en toute sécurité, mais aussi pour " chasser » les phénomènes visés par l'expérience en cours, comme des pluies violentes dans Hymex ou la traînée de condensation d'un autre avion dans TC2 (voir tableau 2). et l'atmosphère pendant Hymex (Ducrocq et al., 2013) a conduit à voler le plus bas possible au-dessus de la mer en évitant les projections d'embruns sur le pare-brise afin de ne pas trop réduire la visibilité pour le pilotage. C'est un des enjeux majeurs de Safire que de remplir ses missions en toute sécurité. Cela passe par un entraînement aérien spécifique pour les pilotes (voir encadré 3). Il faut aussi parfois faire accepter aux scientifiques l'abandon d'une mission dont les risques seraient inacceptables. Sous ces conditions non négociables, c'est donc presque la routine que de maintenir un cap et une altitude, malgré les turbulences rencontrées, pour respecter le plan de vol prévu pour les mesures... tout comme de changer fréquemment de niveau de vol pour effectuer un sondage vertical de l'atmosphère.

L'adaptation permanente du vol aux conditions expérimentales est désormais facilitée par l'existence d'un moyen de communication numérique entre les expérimentateurs à bord et les scientifiques au sol, voire avec d'autres avions de recherche évoluant pour la même mission. Grâce au système Planet $^{3}$, les scientifiques peuvent échanger par chat et recevoir des fichiers, tels que de l'imagerie satellite ou radar, afin de suivre au mieux les évolutions des conditions expérimentales et de les évaluer avec le soutien des équipes terrestres.

2. Ce travail a valu la médaille de bronze du CNRS à Jérémie Vaubaillon en 2013.

3. Développé par la société Atmosphere, en partenariat avec Safire et Météo-France notamment. 


\section{Toute une gamme d'instruments scientifiques}

Une fois la mission scientifique définie et l'avion le plus adapté choisi, une configuration instrumentale est établie (voir l'encadré 4). Dès la date de la campagne connue démarre alors le chronomètre pour tenir les délais impératifs de préparation des instruments de mesure ; alors, selon un retro-planning bien rodé, les équipes de Safire peuvent installer les équipements requis à l'intérieur comme à l'extérieur des avions (tableau 1).

En comparaison avec une station météorologique terrestre, les capteurs embarqués doivent permettre de mesurer des paramètres qui fluctuent beaucoup plus rapidement qu'au sol en raison de la vitesse de vol. La résolution spatiale des mesures étant directement liée à la résolution temporelle, l'échantillonnage doit se faire à des cadences dépassant souvent les cent mesures par seconde. Par ailleurs, l'exposition des capteurs doit prendre en compte le déplacement de l'avion (échauffement cinétique, écoulement perturbé par l'aérodynamique de l'avion...). Nombre d'instruments commerciaux ou développés en laboratoire doivent être adaptés afin de pouvoir rester performants malgré les contraintes thermiques et mécaniques imposées par les conditions de mesure.

\section{Les paramètres atmosphériques de base...}

Systématiquement, les vecteurs de Safire emportent les moyens de mesurer les paramètres atmosphériques les plus classiques : la température de l'air, son humidité, le vent et la pression. Lorsque cela est nécessaire, on ajoute une veine qui permet de prélever en vol des échantillons d'air, distribués en temps réel vers plusieurs appareils d'analyse pouvant provenir de plusieurs laboratoires si besoin.

Pour chaque paramètre de base, l'emploi de plusieurs méthodes de mesure et capteurs à des emplacements différents est souvent nécessaire. Par exemple, des antennes Rosemount ${ }^{\circledR}$ qui protègent les capteurs de température et d'humidité tout en minimisant les effets aérodynamiques indésirables sont placées autour du fuselage, en plusieurs points vers l'avant de l'avion. Cette redondance réduit les incertitudes de mesure. L'humidité,

Tableau 1. Liste condensée des instruments des avions de Safire.

\begin{tabular}{|c|c|}
\hline Paramètres mesurés & Caractéristiques générales, particularités \\
\hline \multicolumn{2}{|c|}{ Radiométrie (mesure du flux de rayonnement électromagnétique) } \\
\hline Flux de rayonnement solaire global & 200 à $3600 \mathrm{~nm}$, vers le haut et le bas \\
\hline Flux de rayonnement infrarouge & 4,5 à $42 \mu \mathrm{m}$, vers le haut et le bas \\
\hline Luminance et température radiative & $\begin{array}{l}\text { Trois canaux infrarouge de largeur } 1 \mu \mathrm{m}: 8,7 ; 10,8 \text {; } \\
12 \mu \mathrm{m} \text { (vers le bas) }\end{array}$ \\
\hline \multicolumn{2}{|c|}{ Microphysique/aérosol } \\
\hline Contenu en eau liquide & $0-10 \mathrm{~g} / \mathrm{m}^{3} ; 0-3 \mathrm{~g} / \mathrm{m}^{3}$ \\
\hline Granulométrie aérosols & $0,06-1 \mu \mathrm{m} ; 0,12-3,12 \mu \mathrm{m}$ \\
\hline Granulométrie gouttelettes nuageuses & $2-50 \mu \mathrm{m}$ \\
\hline $\begin{array}{l}\text { Granulométrie + eau liquide } \\
\text { + vitesse air (10-300 } \mathrm{m} / \mathrm{s})\end{array}$ & 12,5 à $1550 \mu \mathrm{m}$ (2D 64 diodes) ( $25 \mu \mathrm{m}$ de résolution) \\
\hline Granulométrie & $\begin{array}{l}25-800 \mu \mathrm{m} \text { (32 diodes) ; } 200-6000 \mu \mathrm{m} \\
\text { (32 diodes, } 30 \text { classes) }\end{array}$ \\
\hline Détecteur de givre & Sur Falcon 20 \\
\hline Contenu en eau totale & Liquide et solide \\
\hline Lidar rétrodiffusion & Mesure multispectrale et bipolarisation \\
\hline \multicolumn{2}{|c|}{ Thermodynamique } \\
\hline \multicolumn{2}{|l|}{ Pression atmosphérique } \\
\hline Température de l'air & Fluctuations lentes et rapides \\
\hline \multicolumn{2}{|l|}{ Température du point de rosée } \\
\hline Humidité relative & Fluctuations lentes et rapides \\
\hline \multicolumn{2}{|l|}{ Rapport de mélange de la vapeur d'eau } \\
\hline \multicolumn{2}{|c|}{ Dynamique } \\
\hline $\begin{array}{l}\text { Positionnement et vitesse } \\
\text { dans le référentiel terrestre }\end{array}$ & Longitude, latitude, altitude \\
\hline Hauteur & Radio-altimétrie \\
\hline \multicolumn{2}{|l|}{ Altitude, pression } \\
\hline \multicolumn{2}{|l|}{ Vitesse par rapport à l'air, dérapage, incidence } \\
\hline \multicolumn{2}{|r|}{ Chimie } \\
\hline \multicolumn{2}{|c|}{ Concentration en oxydes d'azote $\left(\mathrm{NO}, \mathrm{NO}_{2}\right) \quad$ Chimiluminescence } \\
\hline \multicolumn{2}{|c|}{ Concentration en oxydes de carbone $\left(\mathrm{CO}_{1} \mathrm{CO}_{2}\right)$ Absorption infrarouge } \\
\hline \multicolumn{2}{|c|}{ Concentration en ozone $\quad$ Absorption ultraviolet ( \pm 2 ppbv) } \\
\hline \multicolumn{2}{|c|}{ Taux de dissociation $\mathrm{NO}_{2}$ (haut/bas) $\quad$ Radiométrie ultraviolet-visible } \\
\hline \multicolumn{2}{|c|}{ Divers enregistrements } \\
\hline \multicolumn{2}{|l|}{ Images vidéo vers sol } \\
\hline \multicolumn{2}{|l|}{ Images vers l'avant } \\
\hline Image du radar météo de bord & Falcon 20 \\
\hline
\end{tabular}

variable complexe à mesurer, nécessite de mettre en œuvre des méthodes variées telles que la mesure directe de température du point de rosée ou bien les fluctuations rapides de la quantité de vapeur d'eau. Une mesure directe par un hygromètre capacitif complète le dispositif de façon permanente sur l'ATR 42.

Parmi les paramètres de base, la vitesse du vent est l'un des plus difficiles à calculer, car il n'est accessible qu'indirectement au travers de la mesure de plusieurs autres paramètres. Ainsi, le vent relatif à l'avion - c'est-à-dire les effets de son déplacement dans la masse d'air - est connu dans ses trois dimensions par la mesure de la pression en six points différents : la pression statique sur les parois de l'avion et, à l'avant, la pression totale qui tient compte des effets dynamiques du déplacement dans l'axe de l'avion. Deux mesures différentielles complètent le dispositif : l'une en incidence (hautebasse) et l'autre en dérapage (droitegauche) autour de la prise de pression totale. En raison des mesures doublées, ce sont ainsi sept capteurs de pression, incluant un variomètre, qui permettent de calculer la vitesse de l'air par rapport à l'ATR 42. Pour accéder à la vitesse du vent par rapport à la surface terrestre, il faut composer le vecteur vent relatif à l'avion avec celui du déplacement de l'aéronef, fourni dans chacun des appareils par une centrale inertielle de grande précision.

Des capteurs de rayonnement classiques (pyranomètres) sont aussi disponibles, de façon permanente ou optionnelle, pour accéder au flux dans le domaine visible en bande large montant ou descendant, 


\section{Des campagnes de mesure "sur mesure »}

Au sein de l'unité Safire, les campagnes de mesure se succèdent mais ne se ressemblent pas..

Premièrement, et parce que les avions sont mis à disposition de l'ensemble des laboratoires, ce sont régulièrement de "nouvelles têtes» qui font leur apparition dans les locaux. Il existe pourtant des habitués au nombre desquels on compte les équipes du LA, du Lamp, du Lisa (Laboratoire interuniversitaire des systèmes atmosphériques), du LOA (Laboratoire d'optique atmosphérique), du LPCEE, du LSCE (Laboratoire des sciences du climat et de l'environnement), du Cesbio (Centre d'études spatiales de la biosphère), du Latmos, du CNRM, de la Direction technique de l'Insu et plus récemment de l'Onera. Safire, au travers de ses techniciens et ingénieurs spécialistes de la mesure aéroportée, leur apporte un soutien constant, dès la préparation de la mission.

Deuxièmement, parmi les multiples laboratoires avec lesquels Safire collabore, nombreux sont ceux qui souhaitent faire voler leurs propres instruments en plus de l'équipement de base proposé. Un bureau d'étude interne, reposant sur les compétences d'un ingénieur aéronautique, prépare les dossiers afin d'obtenir leur certification aéronautique. Ce sésame, garant de la conformité et de la sécurité aéronautiques, est indispensable pour autoriser un équipement à voler. Le bureau d'étude peut valider chaque nouvelle configuration très en amont des campagnes, en conformité avec le strict cadre réglementaire aéronautique, mais aussi en fonction des contraintes techniques propres à chaque avion, comme la place et l'énergie disponibles à bord. De nombreux échanges sont souvent

selon leur implantation sur ou sous le fuselage. De même, pour le rayonnement infrarouge en bande large, des pyrgéomètres sont disponibles. Enfin, des radiomètres dirigés vers le bas donnent accès aux températures radiatives des zones visées dans plusieurs bandes spectrales adaptées à l'objet d'étude, notamment les nuages et les aérosols.

Lorsque cela est nécessaire, des caméras vidéo enregistrent des images du vol, vers le pare-brise du cockpit pour visualiser des impacts de glace par exemple, mais aussi dans d'autres directions : il s'agit alors de conserver un «témoin » des situations rencontrées.

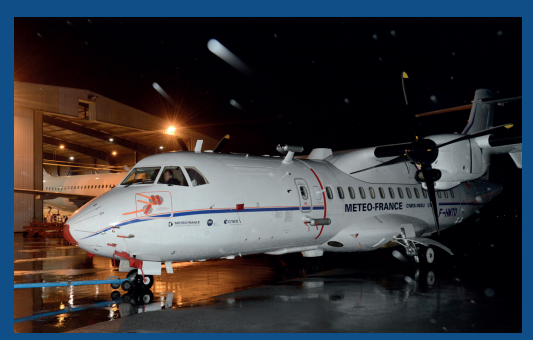

Les exigences expérimentales conduisent à voler par tous temps en tous lieux et à toute heure : ici vol de nuit pour l'expérience Hymex en 2012.

nécessaires pour être fin prêt le jour J. Ce service intégré est très apprécié, car il permet de gagner du temps... et de l'argent.

Enfin, en fonction des phénomènes étudiés, les zones d'investigation varient : par défaut, les avions décolleront de la base de Safire, I'aéroport Toulouse-Francazal ; il offre l'espace et la souplesse nécessaires pour ces opérations de travail aérien très particulières. Dans de nombreux cas, il conviendra de prévoir une base extérieure : cela est vrai par exemple si I'on souhaite étudier le climat d'une région du monde, qu'il s'agisse de l'Afrique de l'Ouest ou de l'Arctique, ou encore si l'on veut être sur un point précis à un instant donné pour suivre la trajectoire d'un satellite lors d'une "Cal/Val »*. L'unité est donc régulièrement appelée à se projeter sur d'autres aéroports dans le monde entier, ce qui implique un important travail de préparation logistique sur le lieu de mission où devra être transporté tout le matériel nécessaire... et le personnel qui travaillera avec.

$\overline{{ }^{*} \text { Calibration ou validation d'une mesure satellitaire }}$ par comparaison à d'autres types de mesure.

Enfin, proches cousines des radiosondes, des «dropsondes » peuvent être larguées depuis le Falcon 20, spécialement équipé de tubes, pour mesurer lors de leur chute vers le sol des profils in situ de pression, température, humidité de l'air et vent, mais aussi d'autres paramètres selon les modèles et les expériences.

\section{Des particules atmosphériques aux processus chimiques...}

La possibilité de mesures à l'intérieur des nuages est une particularité des expériences aéroportées. De nombreux dispositifs permettent de compter les gouttelettes et autres particules les composant. Les chercheurs ont à leur disposition des détecteurs « rustiques » à fil chaud, mais aussi des granulomètres optiques à laser, comme 1'Ultra-high sensitivity aerosol spectrometer (UHSAS) ou le Cloud droplet probe (CDP). Enfin, la veine de prélèvement du Counterflow virtual impactor (CVI) permet de mesurer les propriétés microphysiques et chimiques des aérosols interstitiels à l'intérieur des nuages d'eau liquide, et ainsi d'étudier les interactions aérosols-nuages.

Les processus chimiques dans l'atmosphère sont aussi étudiés grâce aux avions de Safire, qui embarquent des instruments des laboratoires adaptés pour un usage aéronautique. Ces appareils permettent de caractériser en temps réel les concentrations de gaz comme l'ozone $\left(\mathrm{O}_{3}\right)$, le monoxyde et le dioxyde de carbone $\left(\mathrm{CO}, \mathrm{CO}_{2}\right)$, les oxydes d'azote $(\mathrm{NO} x)$ et bien d'autres espèces chimiques. Des photomètres spécifiques permettent la mesure des taux de dissociation du dioxyde d'azote $\left(\mathrm{NO}_{2}\right)$ au-dessus et en dessous de l'avion, donnant ainsi une information sur la photochimie de l'ozone. En complément de ces analyseurs, des appareils encore plus performants provenant des laboratoires utilisateurs sont très souvent installés à bord des avions : il s'agit de différents spectromètres de masse permettant d'obtenir des analyses très précises de nombreuses espèces chimiques.

Chaque année, de nouveaux systèmes font leur entrée dans le parc instrumental de l'unité. En 2015, l'unité s'est dotée d'un analyseur de dioxyde de soufre et d'un lidar qui fournira des profils d'aérosols et de nuages, en dessous comme au-dessus des avions.

\section{La plateforme de mesures aéroportées}

La plateforme de mesures aéroportées est un système intégré de plusieurs instruments microphysiques, optiques et physicochimiques aéroportés. Cette plateforme est dédiée à l'observation in situ des nuages pour l'étude des interactions aérosol-nuagesrayonnement, en particulier dans les nuages en phase glace et/ou en phase mixte constitués de particules nuageuses de formes non sphériques et complexes. Les instruments acquis ont été conçus, modifiés et/ou améliorés par le Lamp 
en collaboration avec les fabricants américains DMT et SPEC Inc. (conception du CPSPD (Cloud particle spectrometer with polarization detection) miniaturisé pour une utilisation sur le Falcon 20 de Safire, optimisation des sondes 2D-S (sonde 2D (bidimensionnelle) stéréo) et CDP-2 pour éviter la fragmentation des cristaux après impact sur l'instrument...). Les instruments ont été complètement renouvelés ces dernières années avec notamment: les imageurs 2D-S (imageur stéréo de cristaux de glace de petites et de moyennes tailles $(10 \mu \mathrm{m}-1,3 \mathrm{~mm}$; jusqu'à 105 images de cristaux par seconde), PIP (Precipitation imaging probe, imageur de gros cristaux précipitants de diamètre $100 \mu \mathrm{m}$ $12 \mathrm{~mm}$ ) et CPI (Cloud particle imager, imageur de particules nuageuses, $10 \mu \mathrm{m}-2,3 \mathrm{~mm}$, à haute résolution de 2,3 $\mu \mathrm{m}$ par pixel) ; les spectromètres optiques rapides FCDP (Fast cloud droplet probe) et CDP-2 pour mesurer les tailles de petites particules nuageuses de 2-50 $\mu \mathrm{m}$, ainsi que le spectromètre optique CPSPD avec mesure supplémentaire de la dépolarisation de petites particules individuelles entre 0,5 et $40 \mu \mathrm{m}$ (capacité de distinction de gouttelettes et petits cristaux dès leur nucléation) et le néphélomètre polaire (unique au monde et entièrement conçu et réalisé au Lamp) pour la mesure de la distribution angulaire de la diffusion par les particules. La plateforme de mesures aéroportées est labellisée « instrument national » par l'Insu depuis 2008.

\section{Quelques instruments plus spécifiques pour l'observation spatiale}

En plus de cette liste d'instruments mis à la disposition de la communauté scientifique, les avions peuvent accueillir les instruments spécifiques développés par certains laboratoires pour améliorer la qualité des mesures ou pour accéder à des variables géophysiques particulières.

Des prototypes d'instruments spatiaux, appelés "démonstrateurs », volent aussi avec Safire, à certaines étapes de leur développement. Une fois les instruments spatiaux embarqués à bord d'un satellite, des campagnes ont lieu pour étalonner et valider leurs données en comparant les mesures reçues de l'espace avec celles effectuées par les démonstrateurs aéroportés. Parmi les instruments développés en étroite collaboration avec Safire, Léandre nouvelle génération (LNG) et Kuros sont présentés comme exemples.

Le lidar Léandre nouvelle génération (LNG) développé par le Latmos (Laboratoire atmosphère milieux et observations spatiales) avec les moyens de la Direction technique de l'Insu est un lidar à haute résolution spectrale qui mesure les profils verticaux des propriétés optiques des aérosols et des nuages dans l'atmosphère. Il est composé d'un laser pulsé qui « tire » 20 fois par seconde à trois longueurs d'onde : 355,532 et $1064 \mathrm{~nm}$. Les impulsions laser sont rétrodiffusées par les particules atmosphériques et les hydrométéores puis captées par un télescope intégré à l'appareil, transformées en signal électrique via un boîtier optique de filtrage et numérisées avant leur enregistrement. La combinaison de trois longueurs d'onde, dans le proche infrarouge, le visible et l'ultraviolet, permet de détecter tous les types de particules atmosphériques : aérosols, gouttelettes d'eau et cristaux de glace. La haute résolution spectrale permet de plus de séparer sans ambiguïté les contributions moléculaire et particulaire à la rétrodiffusion totale, et de restituer les propriétés optiques et radiatives des aérosols. Le lidar LNG est le précurseur du lidar spatial qui sera embarqué sur le satellite Earthcare de l'Agence spatiale européenne.

Le radar doppler Kuros en bande $\mathrm{Ku}$ est conçu pour mesurer le spectre directionnel des vagues (répartition de la hauteur de vagues en fonction de leur longueur d'onde et de leur direction de propagation), ainsi que la vitesse et la direction du vent à la surface de la mer. La qualité des mesures effectuées par le prototype développé par le Latmos avec le soutien du Cnes a pu être améliorée grâce aux vols tests réalisés avec Safire de 2012 à 2014. Le principe de mesure est similaire à celui du radar Swim de la mission spatiale franco-chinoise CFOSAT en cours de développement par le Cnes et l'industriel Thalès (lancement prévu d'ici juin 2018). La mise en œuvre de Kuros au cours de campagnes a également pour objectif de préparer les méthodes d'interprétation des données de la mission CFOSAT.

Certaines campagnes sont spécifiquement conçues pour tester un prototype d'instrument appelé à être embarqué sur un satellite. Ce fut l'objet des campagnes effectuées en liaison avec la constellation de satellites A-Train depuis 2007, qui ont permis de tester à la fois le lidar LNG et l'instrument Osiris (Observing system including polarisation in the solar infrared spectrum); celui-ci préfigure un instrument spatial nommé 3MI qui sera lancé à l'horizon 2021 sur la plateforme satellitaire européenne Metop-SG. Le polarimètre Osiris, développé par le LOA (Laboratoire d'optique atmosphérique), étend les capacités de mesures multi-directionnelles de polarisation de l'instrument Polder vers le moyen infrarouge (jusqu'à 2,2 $\mu \mathrm{m}$ ) afin de mieux caractériser les propriétés microphysiques et radiatives des aérosols et des nuages dans l'atmosphère terrestre.

Tous ces instruments de précision nécessitent un suivi régulier. Ils sont régulièrement entretenus, périodiquement étalonnés en laboratoire et vérifiés en vol, avant chaque campagne et lors de vols d'essais spécifiques. Des campagnes de comparaison permettent l'évaluation mutuelle des instruments embarqués et de ceux des laboratoires utilisateurs. Le survol régulier de points de mesure au sol (observatoires d'altitude pour la plupart) complète le dispositif.

\section{Un traitement des données avant leur analyse scientifique}

L'ultime tâche de Safire lors d'une expérience consiste à fournir aux scientifiques les données collectées. Tous les appareils embarqués sont reliés à une centrale d'acquisition qui effectue un enregistrement numérique de toutes les mesures en les horodatant très précisément. À des vitesses de vol pouvant atteindre $200 \mathrm{~m} / \mathrm{s}$, les échantillons d'atmosphère rencontrés évoluent très rapidement et l'on comprend que la précision de datation soit capitale. Toutes les mesures sont datées à mieux que la microseconde près. Les données étant géolocalisées grâce à une centrale à inertie de précision, elles sont reliées à un solide référentiel spatio-temporel

Souvent, les appareils installés par les laboratoires utilisateurs disposent de leur système d'acquisition autonome. Dans ce cas, une synchronisation des 
Tableau 2. Liste des projets scientifiques ou de recherche et développement pour lesquels Safire a volé.

\begin{tabular}{|c|c|c|c|c|c|c|}
\hline Campagne & Années & Avions & Description & Base départ & $\begin{array}{l}\text { Organisme } \\
\text { référent }\end{array}$ & $\begin{array}{l}\text { Tutelles } \\
\text { participant } \\
\text { au projet }\end{array}$ \\
\hline Capitoul & $\begin{array}{l}2004 \\
\text { et } 2005\end{array}$ & Piper Aztec & Étude de l'îlot de chaleur urbain & Toulouse-Francazal & CNRM & $\begin{array}{l}\text { Météo-France, } \\
\text { CNRS }\end{array}$ \\
\hline Predem & 2005 & Piper Aztec & $\begin{array}{l}\text { Caractérisation des conditions atmosphériques } \\
\text { pour la propagation radar }\end{array}$ & Hyères & DGA & \\
\hline Amma & 2006 & $\begin{array}{l}\text { ATR } 42 \\
+ \text { Falcon } 20\end{array}$ & Étude de la mousson africaine & $\begin{array}{l}\text { Niamey, Niger } \\
+ \text { Dakar, Sénégal }\end{array}$ & $\begin{array}{l}\text { CNRM, } \\
\text { IPSL, OMP }\end{array}$ & $\begin{array}{l}\text { Météo-France, } \\
\text { CNRS, Cnes }\end{array}$ \\
\hline Cops & 2007 & $\begin{array}{l}\text { ATR } 42 \\
+ \text { Falcon } 20\end{array}$ & $\begin{array}{l}\text { Étude de la convection profonde dans } \\
\text { la région des Vosges et de la Forêt noire }\end{array}$ & Baden, Allemagne & $\begin{array}{l}\text { CNRM, } \\
\text { Delft University } \\
\text { of Technology, IPSL }\end{array}$ & $\begin{array}{l}\text { Météo-France } \\
\text { CNRS }\end{array}$ \\
\hline Circle2 & 2007 & Falcon 20 & $\begin{array}{l}\text { Étude des cycles de vie des nuages cirrus. } \\
\text { Validation des satellites Calipso et Cloudsat } \\
\text { par le Lidar Rali }\end{array}$ & Toulouse-Francazal & Latmos & Cnes \\
\hline Carols-Smos & $\begin{array}{l}2007 \\
\text { à } 2010\end{array}$ & ATR 42 & Validation du satellite Smos & Toulouse-Francazal & Latmos & Cnes \\
\hline A-Train & $\begin{array}{l}2007 \\
\text { à } 2014\end{array}$ & Falcon 20 & $\begin{array}{l}\text { Test du lidar LNG et du radiopolarimètre Osiris } \\
\text { (mission Calipso) pour la caractérisation en } \\
\text { profils verticaux des aérosols et des nuages } \\
\text { de l'atmosphère }\end{array}$ & $\begin{array}{l}\text { Toulouse-Francazal } \\
+ \text { Creil }\end{array}$ & LOA, Latmos & Cnes \\
\hline Glori & $\begin{array}{l}2007 \\
\text { à } 2015\end{array}$ & ATR 42 & Télédétection GNSS des surfaces & Toulouse-Francazal & Cesbio & Cnes \\
\hline Adeline & $\begin{array}{l}2007 \\
\text { et } 2008\end{array}$ & ATR 42 & Test d'une sonde aéronautique multifonction & Toulouse-Francazal & Thales Avionics & Météo-France \\
\hline Eucaari & 2008 & ATR 42 & Interaction aérosol-nuage-rayonnement & Rotterdam, Pays-Bas & CNRM, IPSL & $\begin{array}{l}\text { Météo-France, } \\
\text { CNRS }\end{array}$ \\
\hline Flysafe & 2008 & ATR 42 & $\begin{array}{l}\text { Étude des conditions météorologiques dangereuses } \\
\text { pour l'aviation, développement d'instruments } \\
\text { de bord pour l'aviation commerciale }\end{array}$ & Toulouse-Francazal & Thalès & Météo-France \\
\hline Polarcat & 2008 & ATR 42 & Rôle des aérosols et de la pollution anthropique & $\begin{array}{l}\text { Kiruna, Suède }+ \\
\text { Groenland, Danemark }\end{array}$ & $\begin{array}{l}\text { IPSL, Latmos, } \\
\text { Lamp, MPI, } \\
\text { Université de Bruxelles }\end{array}$ & CNRS, Cnes \\
\hline Megapoli & $\begin{array}{l}2009 \\
\text { et } 2010\end{array}$ & $\begin{array}{l}\text { Piper Aztec, } \\
\text { ATR } 42\end{array}$ & Étude du panache de pollution urbaine parisienne & Paris & Lisa & $\begin{array}{l}\text { Météo-France, } \\
\text { CNRS }\end{array}$ \\
\hline Fluxsap & 2010 & Piper Aztec & Mesures infrarouges au-dessus de Nantes & Nantes & IRSTV & \\
\hline Meghatropiques & $\begin{array}{l}2010 \\
\text { et } 2011\end{array}$ & Falcon 20 & $\begin{array}{l}\text { Mesures microphysiques dans la mousson } \\
\text { en Afrique de l'Ouest et en Asie du Sud-Est }\end{array}$ & $\begin{array}{l}\text { Niamey, Niger } \\
+ \text { Gan, Maldives }\end{array}$ & Latmos, Lamp & Cnes \\
\hline I-Wake + Geomad & 2010 & ATR 42 & Turbulence sous l'île de Madère + gravitométrie & Madère, Portugal & $\begin{array}{l}\text { Universités de Porto } \\
\text { et Funchal (Portugal) }\end{array}$ & \\
\hline Simode & 2010 & ATR & $\begin{array}{l}\text { Mesures simultanées radar Storm, radiomètre } \\
\text { Carols et vidéo, dessus de l'océan }\end{array}$ & Brest & $\begin{array}{l}\text { MIO, Latmos, } \\
\text { Ifremer }\end{array}$ & Cnes \\
\hline Bllast & 2011 & Piper Aztec & Turbulence couche limite & Tarbes & CNRM, LA & $\begin{array}{l}\text { Météo-France, } \\
\text { CNRS }\end{array}$ \\
\hline Fennec & 2011 & Falcon 20 & Étude de la dépression thermique saharienne & $\begin{array}{l}\text { Fuerteventura- } \\
\text { Canaries, Espagne }\end{array}$ & Latmos & \\
\hline Draconides & 2011 & Falcon 20 & Suivi d'une pluie de météorites & Kiruna, Suède & IMCCE & \\
\hline Charmex & $\begin{array}{l}2011 \\
\text { à } 2014\end{array}$ & $\begin{array}{l}\text { ATR } 42 \\
+ \text { Falcon } 20\end{array}$ & $\begin{array}{l}\text { Rôle des aérosols et composés chimiques } \\
\text { sur le climat méditerranéen }\end{array}$ & $\begin{array}{l}\text { Avignon, Gênes et } \\
\text { Cagliari, Italie }\end{array}$ & $\begin{array}{l}\text { IPSL, LOA, OMP, } \\
\text { CNRM }\end{array}$ & $\begin{array}{l}\text { Météo-France, } \\
\text { CNRS, Cnes }\end{array}$ \\
\hline Altius & 2012 & ATR 42 & $\begin{array}{l}\text { Instrument atmosphérique embarqué } \\
\text { sur un microsatellite }\end{array}$ & Toulouse-Francazal & $\begin{array}{l}\text { Institut d'aéronomie } \\
\text { spatiale de Belgique }\end{array}$ & \\
\hline Hymex & $\begin{array}{l}2012 \\
\text { et } 2013\end{array}$ & $\begin{array}{l}\text { ATR } 42 \\
+ \text { Falcon } 20\end{array}$ & $\begin{array}{l}\text { Étude des précipitations intenses } \\
\text { dans le bassin méditerranéen }\end{array}$ & $\begin{array}{l}\text { Toulouse-Francazal } \\
+ \text { Montpellier }\end{array}$ & $\begin{array}{l}\text { CNRM/Game, IPSL, } \\
\text { Lamp }\end{array}$ & $\begin{array}{l}\text { Météo-France, } \\
\text { CNRS, Cnes }\end{array}$ \\
\hline Itaac-Corac TC2 & $\begin{array}{l}2012 \\
\text { à } 2014\end{array}$ & Falcon 20 & Étude des traînées de condensation & Toulouse-Francazal & Cerfacs & \\
\hline Libs & 2013 & ATR 42 & $\begin{array}{l}\text { Spectroscopie laser pour caractérisation chimique } \\
\text { de poussières en suspension }\end{array}$ & Toulouse-Francazal & Ineris & \\
\hline Alids & 2013 & ATR 42 & $\begin{array}{l}\text { Test du laser Alids pour mesurer la distribution } \\
\text { granulométrique des gouttes }\end{array}$ & Toulouse-Francazal & IRSN & \\
\hline Hypex & $\begin{array}{l}2013 \\
\text { et } 2014\end{array}$ & Piper Aztec & Imagerie hyperspectrale & Toulouse-Francazal & Onera & Cnes \\
\hline Kuros & $\begin{array}{l}2013 \\
\text { à } 2015\end{array}$ & ATR 42 & $\begin{array}{l}\text { Test du radar Kuros avant mission satellitaire } \\
\text { destinée à surveiller sur les océans, le vent } \\
\text { et les vagues }\end{array}$ & $\begin{array}{l}\text { Toulouse-Francazal } \\
+ \text { Brest }\end{array}$ & Latmos & $\begin{array}{l}\text { Météo-France, } \\
\text { CNRS, Cnes }\end{array}$ \\
\hline Temmas & 2015 & ATR 42 & Imagerie hyperspectrale & Toulouse-Francazal & Onera & \\
\hline Hisa & 2015 & Falcon 20 & Navigation stellaire & Toulouse-Francazal & DGA & \\
\hline Casper & 2015 & ATR 42 & CALNAL satellite & Toulouse-Francazal & Cnes & Cnes \\
\hline HAIC / High IWC & $\begin{array}{l}2014 \\
\text { et } 2015\end{array}$ & Falcon 20 & $\begin{array}{l}\text { Étude des cristaux de glace et teneur en eau } \\
\text { des systèmes convectifs tropicaux }\end{array}$ & $\begin{array}{l}\text { Cayenne, Guyane, } \\
\text { Darwin, Australie }\end{array}$ & Airbus & $\begin{array}{l}\text { Météo-France, } \\
\text { CNRS }\end{array}$ \\
\hline
\end{tabular}




\section{Utilisation du Falcon 20 dans le cadre du projet international HAIC (High altitude ice crystals)}

Au cours de ces dernières décennies, des incidents de type perte de puissance sur les moteurs et/ou anomalies de mesures de vitesse aérodynamique et de température ont été rapportés par les pilotes de ligne au cours de vols à haute altitude. Lors de ces événements, les températures enregistrées étaient pour la plupart inférieures à $-40{ }^{\circ} \mathrm{C}$ et aucun dépôt de glace n'était détecté sur la structure de I'avion. Dans ces conditions, le phénomène de givrage classique par impact de gouttelettes d'eau liquide est exclu. Par ailleurs, le radar de l'avion n'indiquait pas de risque météorologique majeur. En conséquence, la cause des incidents sur les réacteurs et des anomalies d'indication de vitesse ou de température à haute altitude est fort probablement liée à l'ingestion de grandes quantités de microcristaux de glace, difficilement détectables avec les radars embarqués. Ainsi, l'étude des propriétés des cristaux de glace (masse totale de glace dans le nuage contenu en glace, distribution en taille, masse volumique des cristaux de glace, etc.) et des processus responsables de leur formation est de première importance pour la sécurité aéronautique (Lawson et al., 1998 ; Mason et al., 2006). Ces informations sont en effet utilisées par le groupe d'experts international EIWG (Engine icing working group) en soutien à la définition des normes de représentation de l'atmosphère à haute altitude.

\section{Explorer les cristaux de glace à haute altitude}

Les projets HAIC (Dezitter et al., 2013) et High IWC, financés par I'Union européenne, I'EASA (European Aviation Safety Agency) et la FAA (Federal Aviation Administration, États-Unis), et coordonnés par Airbus et la Nasa, ont comme objectifs de contribuer à la révision des normes aéronautiques de définition de l'atmosphère à haute altitude, d'étudier les propriétés des zones à fort contenu en glace des nuages convectifs et de contribuer aux développements technologiques d'instruments de détection et d'évitement de ces zones dangereuses. HAIC a débuté en 2012 avec comme objectifs principaux l'étude des causes d'incident réacteur et de givrage des capteurs de vitesse et de température, le développement de moyens de conformité (moyens d'essais et simulation numérique) et la promotion du développement d'instruments et de méthodes de détection pour faciliter l'évitement des zones de fort contenu en glace. Un partenariat s'est créé entre HAIC et le groupe de travail international HIWC (High ice water content), mené essentiellement par les États-Unis, le Canada et l'Australie.

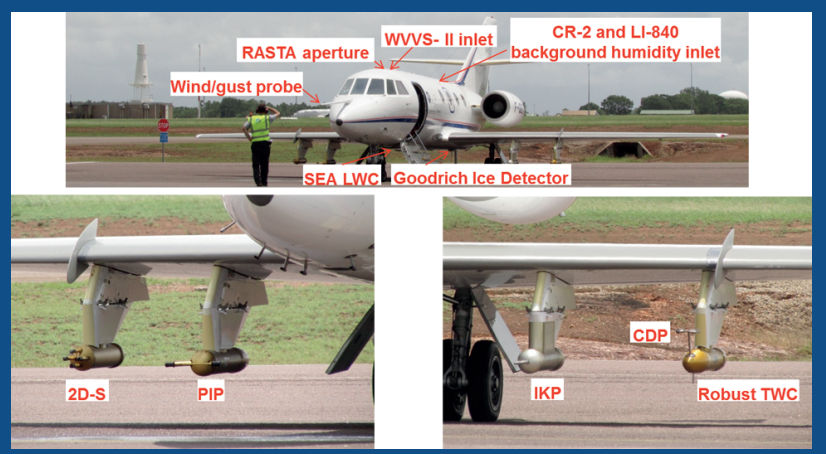

Figure 1. Instrumentation du Falcon 20 avec en particulier un spectromètre optique CDP (Cloud droplet probe), des imageurs optiques de cristaux de glace 2D-stéréo et PIP (Precipitation imaging probe), des instruments de mesure intégrée de contenu en glace IKP (Isokinetic evaporator probe) et Robust, ainsi que le radar de recherche Rasta.
Dans le cadre de cette collaboration internationale, le Falcon 20 de Safire a été équipé avec un ensemble d'instruments sophistiqués pour la caractérisation des propriétés des cristaux de glace, fournis principalement par le Lamp, le Latmos et la Nasa (figure 1). Au total, 120 à 150 heures de vols scientifiques à différents niveaux atmosphériques $(-50,-30$ $-10{ }^{\circ} \mathrm{C}$ ) ont été réalisés dans les nuages convectifs tropicaux, en mettant l'accent sur les zones de fortes concentrations en microcristaux. Ceci facilite un calcul fiable du 99e percentile du contenu total en masse de glace (ice water content, IWC), ainsi que du diamètre massique médian (mass median diameter, MMD), qui fournit une estimation de la taille des cristaux responsables du contenu en glace. IWC et MMD son considérés comme des paramètres microphysiques de première importance en ce qui concerne la définition de l'atmosphère à haute altitude.

Des premières mesures en Australie, complétées ensuite en Guyane

Une première campagne aéroportée HAIC-HIWC d'environ 72 heures de vol a été effectuée depuis Darwin (Australie) en janvier-février 2014 et une seconde campagne de près de 60 heures de vol s'est déroulée à Cayenne (Guyane) en mai 2015. Le Falcon 20 s'est montré capable de voler dans les fortes ascendances de la convection océanique, juste au-dessus des zones de très fortes précipitations (figure 2 , réflectivités radar de couleur rouge et jaune). La figure 3 illustre un cas réel de vol effectué par le Falcon 20 dans un

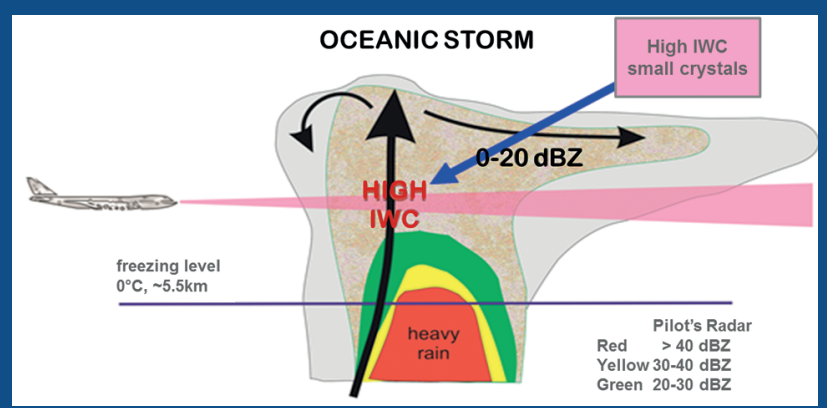

Figure 2. Schéma en coupe verticale d'un passage du Falcon 20 au-dessus d'une cellule convective océanique dans des zones avec fort contenu en en glace (IWC) au-dessus de très hautes réflectivités radar. Source : Strapp et al. (2015)
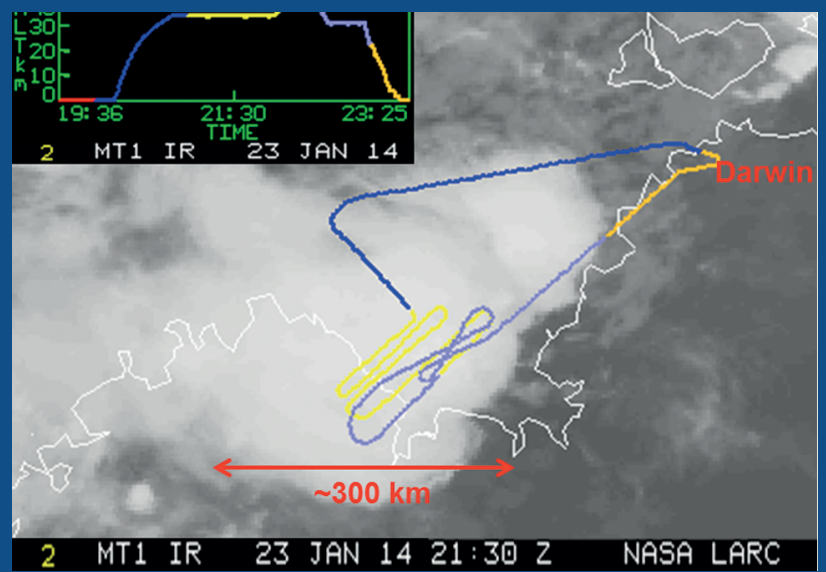

Figure 3. Trajectoire du Falcon 20 dans un système convectif de moyenne échelle dans le golfe Joseph Bonaparte représenté sur une image satellite infrarouge de MTSAT-1R, produite par le Centre de recherche Langley de la Nasa. En haut de la figure : coupe verticale du vol avec code couleur identique aux couleurs de la trajectoire. 


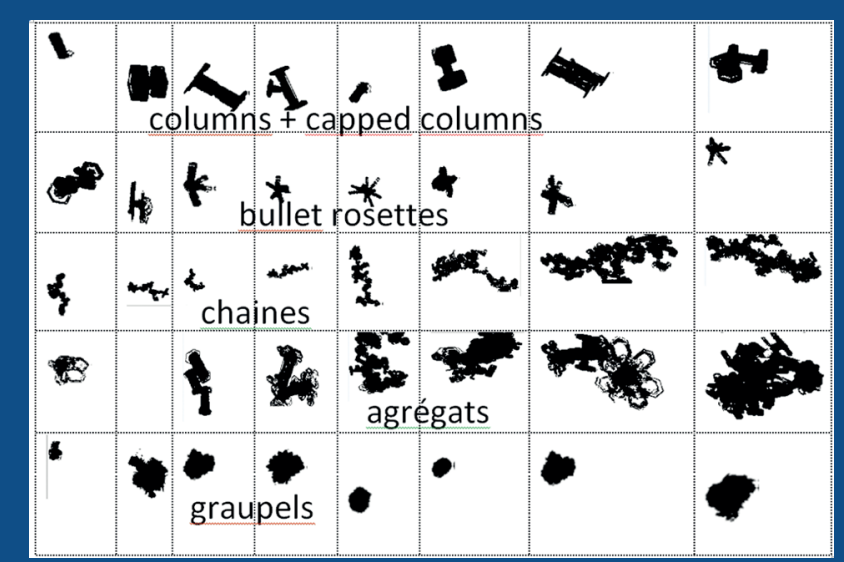

Figure 4. Diversité de cristaux de glace détectés dans des zones de fort IWC de nuages convectifs.

système convectif de moyenne échelle (mesoscale convective system, MCS) d'extension horizontale de plusieurs centaines de kilomètres à la recherche de zones à masses de glace élevées.

L'analyse préliminaire des vols de Darwin montre des valeurs maximales en masse de glace légèrement en dessous de $5 \mathrm{~g} / \mathrm{m}^{3}$ par rapport à une valeur théorique maximale d'environ $7 \mathrm{~g} / \mathrm{m}^{3}$ estimée pour une ascendance adiabatique dans les conditions météorologiques rencontrées à Darwin. Des valeurs supérieures à $2 \mathrm{~g} / \mathrm{m}^{3}$ ont été fréquemment observées sur des distances d'au moins $110 \mathrm{~km}$ durant la plupart des vols effectués. La majorité des zones à forte concentration en glace (observées au moins sur une dizaine de vols) sont constituées de petits cristaux en concentration très importante, échantillonnés dans la convection profonde et jeune. Les formes des cristaux sont dominées par des capped columns, mais on trouve aussi des bullet rosettes, des " chaînes ", des " agrégats » et parfois des graupels (figure 4).

* Le projet HAIC/HIWC a reçu un soutien financier du programme européen HAIC (FP7, numéro de contrat: ACP2-GA-2012-314314), du programme de recherche de I'EASA (numéro de contrat : EASA.2013.FC27), de recherche de I'EASA (numéro de contrat : EASA.2013.FC27),
de la FAA (numéro de contrat $n^{\circ}$ CON-1-1301), de la Nasa, de Boeing Co., de Transport Canada et du BOM.

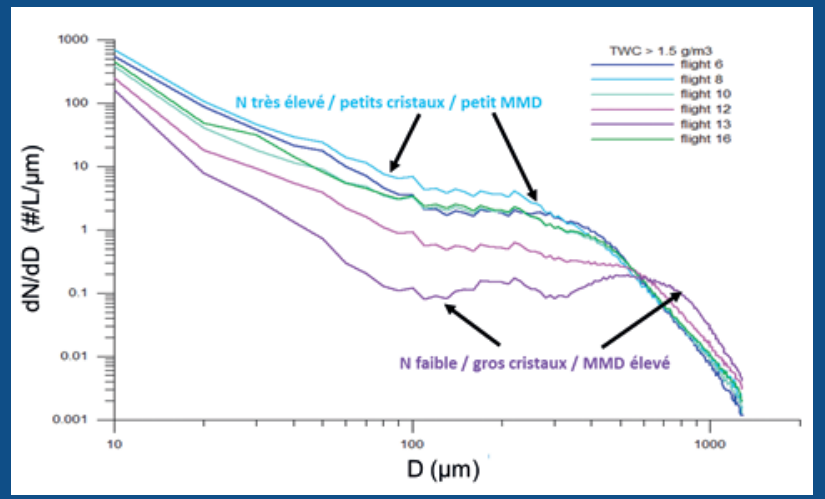

Figure 5. Variabilité des distributions en taille de cristaux des zones avec un contenu de glace élevé observés durant la campagne de mesure HAIC/HIWC

Si on définit la taille des cristaux via leur diamètre équivalent sphérique $D_{\text {eq }}$ (diamètre d'un cercle ayant la même surface que l'image 2D d'un cristal enregistrée par une sonde optique), alors les diamètres massiques médians dans de telles zones atteignent $250-300 \mu \mathrm{m}$. En revanche, au moins deux vols ont été effectués dans des zones de fort contenu en glace, par contre avec des diamètres de cristaux beaucoup plus importants, de l'ordre de 600 $700 \mu \mathrm{m}$, et des concentrations de cristaux assez faibles, montrant que des valeurs élevées de masse de glace peuvent survivre dans des zones de convection plus anciennes sous certaines conditions synoptiques. Cette variabilité importante des diamètres massiques médians (concentrations très élevées de petits cristaux ou concentrations plutôt faibles de plus gros cristaux) est illustrée sur la figure 5, qui montre des exemples de distributions en taille des cristaux obtenus dans des zones à fort contenu en glace pour la campagne $\mathrm{HAIC} / \mathrm{HIWC}^{*}$

Alfons Schwarzenboeck et Delphine Leroy Laboratoire de météorologie physique

Fabien Dezitter et Alice Grandin Airbus Operations $S A S$

Alain Protat Center for Australian Weather and Climate Research, Australie

Julien Delanoë Laboratoire atmosphère, milieux et observations spatiales

Walter Strapp MetAnalytics Inc., Canada

données est effectuée en fonction de l'heure donnée par le système GPS.

Les données font bien entendu l'objet d'un enregisgtrement et d'un stockage sur disque dur, doublé par sécurité sur un autre support. Une fois au sol, les données sont dupliquées vers les systèmes de traitement.

Comme dans toute campagne, un premier traitement sommaire donne aux scientifiques des graphes appelés quicklooks, fournis par Safire peu après le vol. Le traitement en profondeur, en filtrant les mesures et en corrigeant les erreurs de mesure identifiées, établira les paramètres physiques expérimentaux à partir des valeurs instrumentales brutes. Ces traitements qui peuvent durer plusieurs mois sont réalisés en général soit par le $\mathrm{CNRM}^{4}$ en collaboration étroite avec Safire, soit par les laboratoires utilisateurs eux-mêmes. L'étude scientifique de l'expérience peut alors commencer pour les équipes de recherche.

\section{5-2015: les campagnes de Safire}

Au cours de la décennie passée, l'activité de Safire a connu un rythme soutenu comme en atteste le tableau 2 qui résume l'ensemble des campagnes auxquelles a pris part l'unité. Les contributions de ces expériences à l'amélioration des connaissances scientifiques ont souvent été déterminantes et ne peuvent toutes être détaillées. À titre d'exemple, nous illustrons le propos par deux campagnes récentes, portant sur la microphysique (encadré 5) et la chimie atmosphériques (encadré 6).

4. Centre national de recherches météorologiques Groupe d'études de l'atmosphère météorologique, équipe GMEI/TRAMM : traitement et analyse de mesures météorologiques du Groupe météorologie expérimentale et instrumentale. 


\section{Megapoli-Paris : l'ATR 42 complémentaire du dispositif d'observations au sol}

Le projet européen du 7e PCRD Megapoli (Megacities: emissions, urban, regional and global atmospheric pollution and climate effects, and integrated tools for assessment and mitigation, 2008-2011) a traité de l'impact des mégapoles sur la qualité de l'air et la composition chimique de la troposphère. Une campagne d'envergure a été mise en œuvre au cours de l'été 2009 en Île-de-France, région considérée comme un exemple de mégapole de type tertiaire située à une latitude tempérée. L'objectif était une meilleure quantification des sources primaires et secondaires de l'aérosol organique (AO). Un ensemble important et novateur d'instruments a été déployé par les partenaires français et européens (consortium de dix équipes) sur trois sites fixes urbain et péri-urbain, sur des plateformes mobiles et sur l'avion de recherche ATR 42 . Ce dispositif a permis de caractériser la composition chimique des particules, leurs propriétés physico-chimiques, leurs précurseurs gazeux, ainsi que la structure verticale des basses couches de l'atmosphère. La cohérence des mesures obtenues a été testée au cours d'un exercice d'inter comparaison (figure 1) visant à évaluer l'ensemble des instruments.

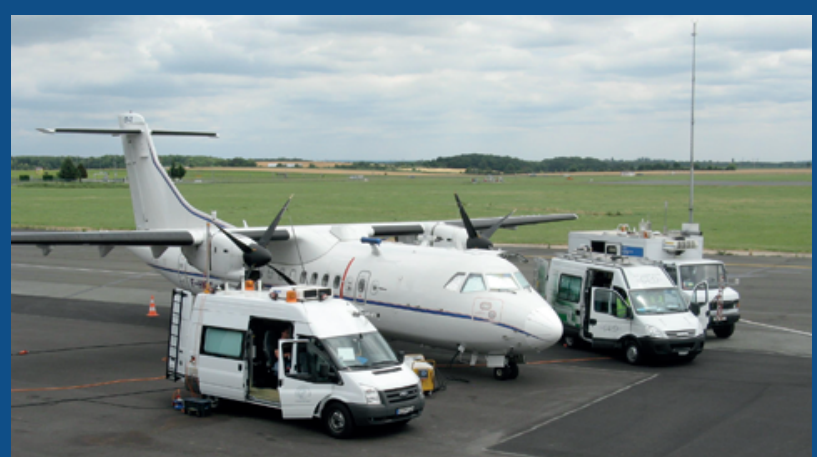

Figure 1. Les plates-formes mobiles et l'ATR42 en intercomparaison.

Améliorer linventaire des émissions des polluants gazeux et particulaires

Un premier axe d'étude a été d'évaluer l'inventaire régiona des émissions de certains polluants gazeux et particulaires (oxydes d'azote NOx, carbone suie BC, composés organiques volatils $\mathrm{COV}$ ), considéré comme présentant encore de larges incertitudes. Une des méthodes consiste à déterminer des rapports de concentrations $\Delta[X] / \Delta[\text { traceur }]^{*}$ à partir d'analyses de corrélation entre les observations et à les comparer aux mêmes rapports extraits de l'inventaire, afin de s'affranchir des effets de la dilution. Une des conditions est la représentativité spatiale des observations qui, limitées à un ou deux sites de surface, peut être sujette à caution compte tenu de I'hétérogénéité de la distribution des émissions (Borbon et al., 2013 ; Petetin et al., 2015). La mesure aéroportée, plus intégrative, permet de lever cette contrainte d'échelle. Un exemple est donné sur la figure 2 dans le cas du toluène composé organique volatil mono-aromatique et un des précurseurs de l'aérosol organique secondaire (AOS), détecté par le spectromètre de masse à transfert de protons communautaire (PTR-MS) embarqué. Dans l'exemple, les rapports à l'émission $\Delta[$ toluène $] / \Delta[\mathrm{CO}]$ ont été établis dans le panache pour deux vols de l'ATR 42 dans les secteurs nord et

* où $X$ est le composé étudié (comme par exemple le toluène dans la figure 2) le traceur est un composé inerte chimiquement à l'échelle de temps de transport du panache permettant de s'affranchir des effets de la dilution du panache; $X$ peut être par exemple le monoxyde de carbone (CO) ou le carbone panache
suie $(B C)$ sud-ouest de l'île-de-France. La similitude de ces rapports avec ceux issus des stations au sol a permis de justifier l'utilisation des rapports de surface pour l'évaluation des inventaires. Les résultats ont montré que, même pour des mégapoles de latitudes moyennes tempérées, les erreurs sur les inventaires des émissions persistaient parfois jusqu'à un facteur 4 pour les composés organiques volatils (Borbon et al., 2013).
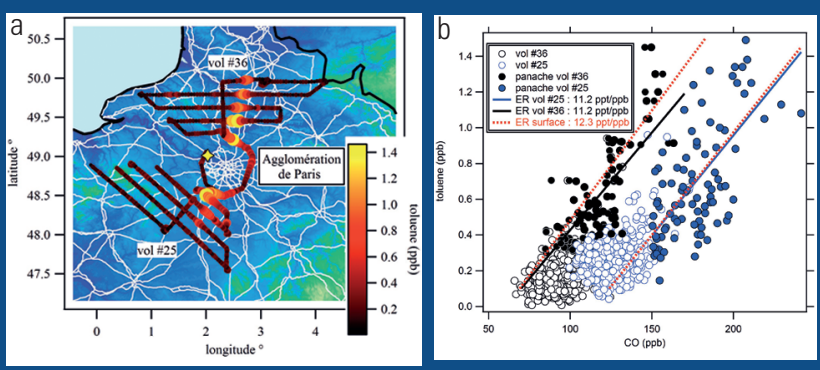

Figure 2. a) Trajectoires des vols 25 (01/07/2009) et 36 (25/07/2009) colorées par les concentrations en toluène. b) Rapports à l'émission (ER) déterminés à partir des pentes des droites de corrélation toluène vs CO dans le panache de pollution de Paris au cours des deux vols et comparaison avec celui calculé à partir des observations de surface, ER surface (site du Laboratoire d'hygiène de la ville de Paris, XIII arrondissement).

\section{À la recherche des aérosols organiques secondaires}

Un deuxième axe d'étude a porté sur la production de l'AOS dans le panache parisien (Freney et al., 2014). L'ATR 42 a permis de suivre cette production au fur et à mesure que le panache s'éloignait de l'agglomération, à partir des mesures couplées de I'AMS (Aerosol mass spectrometer), du PTR-MS, de MONA (Measurement of nitrogen oxides on aircraft) et de I'analyse des rapports $\Delta[\mathrm{AO}] / \Delta[\mathrm{CO}]$ en fonction de l'âge photochimique de la masse d'air. L'avion a pu échantillonner plusieurs fois un même panache à différents stades d'évolution chimique, avec la flexibilité nécessaire au regard de la dynamique atmosphérique, les régimes de vent déterminant la direction de propagation du panache ; cette flexibilité est très limitée si l'on considère seulement un dispositif de mesure à la surface. Le suivi a montré que la production photochimique de l'AOS était encore active à environ $150 \mathrm{~km}$ du centre de l'agglomération.

Ces deux exemples montrent bien l'apport très complémentaire de l'avion sans lequel le dispositif de surface, seul, n'aurait pu permettre d'aborder les questions des émissions des composés gazeux et particulaires et du devenir de la matière organique dans le panache d'une mégapole comme Paris. L'analyse des observations au cours de la campagne Megapoli a abouti à des résultats importants en permettant d'évaluer les inventaires des émissions de polluants d'intérêt et de montrer que les émissions de la mégapole parisienne contribuent à la formation de l'aérosol organique à l'échelle régionale. Ces observations ont été par ailleurs très précieuses pour l'évaluation des modèles de qualité de l'air, en particulier pour la prévision de l'aérosol organique en atmosphère urbaine qui constitue aujourd'hui un enjeu majeur.

Agnès Borbon, Aurélie Colomb, Evelyn Freney, Alfons Schwarzenboeck Laboratoire de météorologie physique

Matthias Beekmann Laboratoire interuniversitaire des systèmes atmosphériques 
Les vols de mesure de Safire concernent aussi le domaine de la formation. Ainsi l'ATR 42 embarque régulièrement des étudiants de l'École nationale de la météorologie (MétéoFrance/INP Toulouse) et de l'Université Paul-Sabatier (Toulouse) pour une classe volante sur l'instrumentation aéroportée ; l'unité a également participé aux écoles d'été d'Eufar en 2007 à Iasi (Roumanie) et en 2010 à Hyères, ce qui a permis à plus de 50 jeunes scientifiques européens de monter à bord de l'ATR 42. Au service de la communauté scientifique, Safire propose aussi aux laboratoires des vols de comparaison et d'étalonnage de capteurs, que ce soit en microphysique ou en chimie. Enfin, il faut rappeler le rôle de l'ATR 42 et du Falcon 20 dans le dispositif gouvernemental mis en place lors de l'éruption du volcan islandais Eyjafjallajökull en 2010, pendant laquelle ces avions ont participé « au pied levé » à un diagnostic fin de la présence de cendres dans l'espace aérien.

\section{Bilan et perspectives}

La France s'est dotée de moyens conséquents pour couvrir une vaste palette de besoins en recherche environnementale aéroportée. Depuis la création de l'unité en 2005, plus de 3000 heures de vol scientifique ont été accomplies, au bénéfice de plus de 500 chercheurs. Plus de trente laboratoires français et à peu près autant de laboratoires ou organismes de recherche étrangers ont bénéficié des prestations de Safire. L'étendue des domaines d'emploi et la variété des utilisateurs montrent la pertinence de l'organisation et des moyens de Safire, ainsi qu'une adaptabilité à des demandes parfois très éloignées des intentions initiales de ses concepteurs.

On l'a compris, l'activité de Safire se renouvelle régulièrement. Aussi, les opérations aériennes doivent souvent sortir des sentiers battus des lignes commerciales et nécessiter une préparation complexe. Quant au produit fini, les données scientifiques, leur fourniture par l'unité requiert des traitements adaptés à chaque campagne. Bref, dans un contexte très normé par les règles aéronautiques et les exigences de la rigueur scientifique, Safire doit s'adapter sans cesse et proposer du « sur mesure ».

Si la flotte actuelle a su s'adapter à l'évolution des techniques de mesure, les principaux défis à relever consistent à définir les besoins futurs en matière de vecteurs aéronautiques, en intégrant les drones de taille moyenne (environ $5 \mathrm{~kg}$ de charge utile et $8 \mathrm{~h}$ d'autonomie) dans la réflexion. Dès à présent, on anticipe le renouvellement de l'appareil le plus ancien, le Falcon 20, en inscrivant les futurs moyens dans le contexte européen. Les besoins à venir des scientifiques sont appréhendés au travers d'une large consultation des laboratoires, utilisateurs ou susceptibles de l'être.

À la veille de ces échéances capitales, Safire peut compter à la fois sur l'engagement de la communauté scientifique et le professionnalisme de ses personnels pour continuer à servir aux mieux les intérêts scientifiques.

\section{Bibliographie}

Borbon A. et al., 2013. Emission ratios of anthropogenic volatile organic compounds in Northern mid-latitude megacities: Observations vs. emission inventories in Los Angeles and Paris. J. Geophys. Res. Atmos., 118, 2041-2057.

Brenguier J.-L., Ancellet G., 2003. Les avions instrumentés pour la recherche atmosphérique. La Météorologie, 40, 68-73.

Chalon J.-P. et al., 1998. Les avions de recherche atmosphérique et de télédétection ; bilan et perspectives. La Météorologie, 22, 14-44

Chazette P. et al., 2012. French airborne lidar measurements for Eyjafjallajökull ash plume survey. Atmos. Chem. Phys., 12, 7059-7072.

Dezitter F. et al., 2013. HAIC - High altitude ice crystals. Proceedings of the 5th AIAA Atmospheric and Space Environments Conference. doi: 10.2514/6.2013-2674

Ducrocq V. et al., 2013. HyMeX, les campagnes de mesures : focus sur les événements extrêmes en Méditerranée. La Météorologie, 80, 37-47.

Freney E.J., et al., 2014. Characterizing the impact of urban emissions on regional aerosol particles; airborne measurements during the MEGAPOLI experiment. Atmos. Chem. Phys., 14, 1397-1412.

Lawson P.R. et al., 1998. Cloud particle measurements in thunderstorm anvils and possible weather threat to aviation. J. Aircraft, 35, 113-121.

Mason J.G. et al., 2006. The ice particle threat to engines in flight. 44th AIAA Aerospace Sciences Meeting, Reno, Nevada, 9-12 January 2006, AIAA-2006-206.

Petetin H. et al., 2015. Evaluating BC and NOx emission inventories for the Paris region from MEGAPOLI aircraft measurements. Atmos. Chem. Phys., 15, 9799-9818.

Strapp J.W. et al., 2015. The high ice water content (HIWC) study of deep convective clouds: science and technical plan. FAA Report DOT/FAA/TC-14/31, in press.

\section{Sites internet}

www.safire.fr www.eufar.net 